\title{
Long-Term Disease Control of a Non-Operable Neuroendocrine Tumor of the Lung with Lanreotide: A Case Study
}

\author{
F. Van Fraeyenhove ${ }^{a} \quad$ E. De Droogh ${ }^{b} \quad$ N. Meireson ${ }^{c}$ \\ D. Galdermans ${ }^{b} \quad$ C. Goor ${ }^{c} \quad$ F. Van Acker ${ }^{d} \quad$ C. Mattelaer ${ }^{e}$ \\ V. De Ruyter ${ }^{f} \quad$ D. Schrijvers ${ }^{a}$ \\ Departments of ${ }^{a}$ Medical Oncology, ${ }^{b}$ Pneumology, ${ }^{\mathrm{c}}$ Radiotherapy, ${ }^{\mathrm{d}}$ Nuclear \\ Medicine, and ${ }^{\mathrm{e}}$ Pathology, Ziekenhuisnetwerk Antwerpen, Antwerp, and \\ ${ }^{f}$ Medical Department IPSEN NV BeLux, Merelbeke, Belgium
}

\section{Key Words}

Neuroendocrine tumor · Carcinoid · Lung

\begin{abstract}
Bronchopulmonary neuroendocrine tumors (NETs) are malignant tumors that represent approximately $20 \%$ of all lung cancers. The therapeutic option for advanced or metastatic bronchopulmonary NETs is mainly palliation of symptoms; options need to be individualized and, therefore, rely on the knowledge of multidisciplinary teams. Somatostatin analogs have been widely used in NETs for control of hormonal syndromes and are currently under evaluation for their antiproliferative activity. Here, we present a case of NET of the lung, for which we achieved long-term disease control with a treatment comprising the somatostatin analog lanreotide Autoge ${ }^{\circledR}$ in a patient with limited therapeutic options due to considerable comorbidity, while preserving his quality of life.
\end{abstract}

\section{Introduction}

Neuroendocrine tumors (NETs) represent a spectrum of rare tumors originating from diffuse endocrine cells throughout the body. In the USA, NETs comprise $0.73 \%$ of all diagnosed cancers, with an incidence of about 5 cases/100,000/year [1].

The most prevalent of these (55\% of NETs) are in the gastrointestinal tract; however, about $30 \%$ of all NETs originate in the lung [considering only typical carcinoids (TCs) and atypical carcinoids (ACs)] [2].

Dr. Frank Van Fraeyenhove 
Since NET is a rare disease, data from randomized clinical trials addressing questions of diagnosis, treatment, and therapy are limited. Some groups such as the European Society for Medical Oncology (ESMO), the National Comprehensive Cancer Network (NCCN), and the European Neuroendocrine Tumor Society (ENETS) have formulated guidelines on these issues. Most guidelines are consensus texts and few recommendations reach level A evidence. While NETs in most organs arise from the same group of neuroendocrine cells, NETs of the lung represent a specific subgroup comprising different entities. Thus, the available data on gastrointestinal NETs cannot simply be extrapolated to the lung. Case reports are an important tool for directing future research and current treatment strategies for rare diseases.

Here, we present a case of NET of the lung for which we achieved long-term disease control in a patient with limited therapeutic options due to considerable comorbidity. The patient's quality of life was preserved.

\section{Case Report}

A 65-year-old patient was admitted to hospital (summer 2009) to receive an implantable cardioverter defibrillator (ICD) for ischemic coronary disease and ischemic cardiomyopathy with a left ventricular ejection fraction of $40 \%$. The patient had previously received a drug-eluting coronary stent for acute myocardial infarction in early 2009. The patient suffered from dyslipidemia, type 2 diabetes mellitus with existing end organ damage (atherosclerosis, diabetic nephropathy resulting in end stage renal disease, and retinopathy), and stage 2 [Global Initiative for Chronic Obstructive Lung Disease scaling system (GOLD)] chronic obstructive pulmonary disease (COPD). His chronic medication consisted of acetyl salicylic acid, clopidogrel, molsidomine, ranitidine, lisinopril, bisoprolol, metformin, insulin, simvastatin, tiotropium inhaler, salmeterol, and fluticason inhaler

Routine pulmonary X-ray showed a mass in the upper lobe of the left lung. ICD implantation was complicated by an acute episode of ventricular arrhythmias and bronchospasms. In further work-up, a bronchoscopy was performed and again complicated by severe bronchospasm, arrhythmia, and an acute hypertensive crisis, resulting in acute pulmonary edema. The patient was admitted to intensive care to receive supportive therapy and overcame this acute incident. Due to the complications during bronchoscopy, the investigation was inconclusive.

Given that the patient had a relatively good quality of life before this acute episode (Karnofsky score 70/100), further investigations were planned. Positron emission tomography-computed tomography (PET-CT) confirmed a metabolically active lesion in the upper lobe of the left lung $(4 \mathrm{~cm})$, extending to within the proximity of the left hilar region, compatible with a neoplastic process (fig. 1a). There were no pathologic lymph nodes in the region, or distant metastasis.

The diagnosis was impeded by the fact that no lesions could be visualized during the bronchoscopy and a blind biopsy and lavage did not show any abnormalities. Further bronchoscopy was refused due to the two episodes of cardio-respiratory distress during the previous invasive procedures. A CT-guided biopsy could not be performed because the patient was on antiaggregant therapy, which was mandatory for a minimum of 1 year after receiving a drug-eluting stent. The risk of a pneumothorax during such a procedure was substantial due to the anatomic localization of the lesion. This was not considered an acceptable risk given the underlying pulmonary and cardiovascular disease.

The case was reviewed at a multidisciplinary tumor board. A neuroendocrine tumor was suspected based on the two acute episodes during the ICD implantation and bronchoscopy procedures, indicating a potential carcinoid crisis. An octreotide scan showed a tumoral mass accumulating in the radiotracer, confirming the presence of somatostatin receptors in the neoplastic lesion (fig. 1b). In a 24-hour urine collection, serum chromogranin A ( $\operatorname{CgA}$ ) was elevated ( 3 times upper normal limit) and 5-hydroxyindole acetic acid (5-HIAA) was normal 
Surgical resection was not performed due to the above-mentioned complications. The patient was referred for radiation therapy at the tumoral site. A dose of $60 \mathrm{~Gy}$ was delivered in 30 sessions using 3D-conformal radiotherapy (from November 12 to December 24, 2009). The patient received $90 \mathrm{mg}$ lanreotide Autoge ${ }^{\circledR}$ every 4 weeks during and up to 1 month after completion of the radiation therapy. The patient tolerated the treatment well and started to become active once again in social life and in domestic duties.

Between December 2009 and May 2010, the pneumologist noted six exacerbations of the known COPD, and a further two episodes requiring hospitalization (GOLD stage 3). The patient's Karnofsky score was 60/100. A radiological evaluation showed infiltrates at the site of the primary tumor, expanding towards the mediastinum. Potential causes were changes secondary to the radiation treatment, infection, or progressive disease. The patient did not show signs of infection (the medical history was negative, there was no fever and no elevation of inflammatory parameters in the patient's serum). A new octreotide scan revealed new lesions in the upper lobe of the left lung and activity in the irradiated mass [5.5 cm width; Response Evaluation Criteria In Solid Tumors (RECIST)] (fig. 2a). There was no evidence of distant metastasis. Lanreotide Autogel was reintroduced $(90 \mathrm{mg}$ every 4 weeks). Over the following 6 months, there was a significant decrease in COPD exacerbations (GOLD stage 2) and performance status improved (Karnofsky score 80/100).

Treatment with lanreotide Autogel was continued. In the winter of 2010, the patient presented with an episode of hemoptysis. Common Terminology Criteria for Adverse Events (CTCAE) scoring revealed grade 1 toxicity. This was not attributed to the use of lanreotide, but was probably due to changes in the large pulmonary bronchi following radiation treatment. CT imaging documented a stable disease (RECIST criteria [3]); absolute measurements of the lesions showed a slight decrease in the diameter of the upper lobe of the left lung. The patient was treated with tranexamic acid for the hemoptysis, and lanreotide Autogel was continued at $120 \mathrm{mg}$ per month.

At the time of the patient's last visit, a slight increase in episodes of hemoptysis from December 2011 was noted, without impacting daily activities (CTCAE grade 1). Imaging (June 2012) showed some tumor growth $(6.0 \mathrm{~cm}$ longest diameter) (fig. $2 \mathrm{~b}$ ). The disease was stable according to RECIST criteria when compared to imaging at the time of relapse, with the patient scoring $80 / 100$ on the Karnofsky scale.

\section{Discussion}

NETs of the lung arise from enterochromaffin cells of the aerodigestive tract. They comprise different tumor types sharing a number of neuroendocrine features and include small cell lung carcinoma (SCLC), large cell neuroendocrine carcinoma (LCNEC), TCs, and ACs.

The prognosis of TCs is good and these indolent tumors have a low rate of recurrence after adequate resection, even if metastasis in regional lymph nodes is present. ACs are less common, are considered intermediate in grade, and are associated with a poorer prognosis. Currently, the work-up and identification for surgery are the same as for non-SCLC. The role of adjuvant chemotherapy for these tumors has not been defined [4]. Distant metastasis is rare (7\%). A 5-year survival rate of $90 \%$ has been reported for TC and 60\% for AC. These figures differ from those for LCNEC and SCLC, which are associated with a poor prognosis and 5-year survival rates of $<35 \%$ for LCNEC and $5 \%$ for SCLC $[5,6]$.

A number of strategies have been proposed for diagnosis and treatment by pulmonary and oncologic consensus panels. Histological classification of NETs and adequate staging of the tumor are key to defining an optimal therapeutic strategy. Various histological classifications and definitions have been proposed [7]. The World Health Organization classification system was proposed in 2004 and provides standard 
definitions concerning the diagnosis and management of NETs of the lung (table 1). Recent histology, immunohistochemical and molecular studies suggest that the lung carcinoid group is distinct from the more malignant LCNEC and SCLC groups $[8,9]$. Therapeutic options thus depend on the subtype and stage of the NET.

Figure 3 represents simplified diagrams for diagnostic and therapeutic approaches [7]

The major challenge in the case presented here was obtaining histological evidence for diagnosis of NET. Indirect evidence, including the clinical presentation, CgA levels, and octreotide scan, gave a good indication of the diagnosis.

We used PET-CT and octreotide scans to detect tumoral lesions. A review of the literature does not reveal any major differences in sensitivity between these methods (up to 90\%). An octreotide scan detects tumors expressing serotonin receptors, with the uptake of octreotide supporting the potential use of therapeutic agents targeting these receptors. However, tumors not expressing these receptors, such as ACs, would be missed by an octreotide scan $[7,10]$.

Due to the risk of complications, we did not carry out further invasive procedures to acquire histological material, and therapy was initiated without definite pathology. Therapeutic options were limited in our patient and the disease was localized. We therefore chose radiation therapy with curative intent. Although this would not be the treatment of choice in fit patients, ESMO practice guidelines describe external radiotherapy as an option for selected cases in which surgery cannot be performed [6]. Delineation of the target volume and subsequent dose distribution could not provide optimal coverage of the target volume towards the mediastinum, due to the proximity of a pre-existing weakened heart. This is most likely the reason for relapse in proximity of the mediastinum. Bronchopulmonary NETs are also generally considered to be more resistant to treatment than the more common lung neoplasms (e.g. carcinoma of the lung) [5, 7]. Radiotherapy can induce inflammation in the irradiated area; thus, lanreotide Autogel was administered during radiation treatment to avoid carcinoid crisis. This was discontinued thereafter, since radiation treatment was performed with curative intent. There is no evidence in the literature that adjuvant treatment in bronchopulmonary NETs is beneficial, thus this was not indicated $[6,11]$.

\section{Follow-Up}

NET diagnosis could not be confirmed at the time of relapse due to the patient being on antiaggregation therapy following previous insertion of drug-eluting stents. The patient showed clinical deterioration with COPD exacerbations, and an octreotide scan showed high uptake of octreotide. Lanreotide Autogel was therefore reintroduced with good results. We did not choose to use chemotherapy because response rates in NETs of the lung are low and the toxicity profile in a diabetic patient with COPD, heart failure, and impaired renal function would not be favorable [6].

The majority of patients with bronchopulmonary NETs are symptomatic, although up to $45 \%$ of cases may exhibit no signs. The most common symptoms are cough (32\%), pneumonia (26\%), and hemoptysis $(24 \%)$ [5, 7]. Our patient experienced an increase in COPD exacerbations, which may be explained by the production of serotonin and other neuropeptides, by changes induced in the lung following 
radiotherapy, or by disease progression with irritation of central bronchi by tumor nodules. The fact that the use of lanreotide Autogel abruptly stopped COPD exacerbations in this case suggests that blocking the serotonin receptors had major benefits for this patient.

During the course of the disease, $\operatorname{CgA}$ levels were measured. This is a sensitive marker of NETs, but may be normal in small lesions. It can also be elevated in a number of situations, including in the use of proton pump inhibitors (CgA levels $\times 2-3$ ), steroid use, and renal impairment (in end stage renal disease, CgA levels are raised up to $\times 10$ ) [12]. Octreotide scan and imaging were used for diagnosis, while only imaging was used for disease follow-up in this patient.

\section{The Choice of the Somatostatin Analog Lanreotide}

Lanreotide Autogel was selected among commercially available somatostatin analogs (SSAs) based on its action in symptom control and its potentially antiproliferative effect [13]. There is currently no reported difference in clinical outcome between the different types and formulations of SSAs. Additionally, practice guidelines from medical societies (NCCN, ESMO, and ENETS) do not differentiate between them for use in this indication $[6,11,14]$. Recent in vitro studies, however, have shown that NET cell lines, from different sites and arising from different types of neuroendocrine cells, respond differently to individual SSAs. One study showed substantially different cell surface expression profiles for somatostatin and dopamine receptors in lung and gastrointestinal cell lines [15]. Being differently coupled to microtubule-associated protein kinase (MAPK)/extracellular signal-regulated kinase (ERK) and c-Jun amino-terminal kinase (JNK) signaling pathways, these receptors can differently influence tumor cell proliferation [15]. In that study, SSAs and somatostatin/dopamine chimeric compounds exhibited different inhibitory effects on cell lines of different organs (lung vs. gastrointestinal), as well as on cell lines from the same organ (lung: TC vs. AC). Lanreotide was the most effective inhibitor in the AC and small intestine carcinoid cell lines. In contrast, none of the tested molecules in the study, despite the expression of somatostatin receptors, inhibited the proliferation of TC. Synthetic derivatives of somatostatin, such as octreotide, are effective in inhibiting the biosecretion of bioactive products, but have been disappointing as antiproliferative agents in vivo. The reasons for this are unknown. However, these findings suggest that secretory regulation is an intrinsic property of NET cells, whereas the proliferative drive may involve a different mechanism. Thus, rather than adopting a global strategy based purely upon histological characterization or classification, therapeutic strategies should be individualized according to the receptor profile of a specific lesion. Until such data are available, one can only adapt clinical practice according to experience.

This case suggests that lanreotide can control the disease. Following a relapse observed 6 months after radiotherapy with curative intent and the reintroduction of lanreotide Autogel treatment, objective scoring using the RECIST criteria showed disease stabilization for up to 2 years. One could conclude that current treatment not only has an effect on symptom control, but also has an impact on disease progression. These data are in line with findings suggesting an antiproliferative effect of certain SSAs in specific (lung) NETs [13]. We are unfortunately unable to match the histological diagnosis (AC) as we have been unable to obtain tissue samples from our patient. 


\section{Conclusion}

This case shows that, even in patients with considerable comorbidity, adequate treatment can promote survival with good quality of life in the absence of side effects from active treatment. Besides excellent symptom control, this patient had fair overall survival when using lanreotide, given the fast relapse of disease (within 6 months of radiotherapy with curative intent). This and other similar cases treated at our institute with similar results support the use of lanreotide in cases of lung NETs. Pending results of further research, we would encourage new case reports to confirm or refute the use of lanreotide in lung NETs, particularly as a potential active treatment line in carcinoids of the lung. Given the sporadic occurrence of lung NETs, centralization would be recommended to ensure standardized treatment by experienced physicians and to create the opportunity for further research in this specific pathology.

\section{Acknowledgements}

We thank Juliette Gray (XPE Pharma and Science, on behalf of IPSEN NV BeLux) for editorial assistance.

Table 1. Histological classification of NETs of the lung [6]

\begin{tabular}{|c|c|c|c|}
\hline Tumor type & Histological findings & Immunohistochemistry & $\begin{array}{l}\text { Malignancy } \\
\text { grading }\end{array}$ \\
\hline $\mathrm{TC}$ & $\begin{array}{l}\text { carcinoid architecture; } \\
<2 \text { mitoses/10 HPF }\end{array}$ & $\begin{array}{l}\text { CgA; synaptophysine; } \\
\text { NSE; expression of SR }\end{array}$ & $\begin{array}{l}\text { grade } 1 ; \\
\text { low }\end{array}$ \\
\hline$\overline{\mathrm{AC}}$ & $\begin{array}{l}\text { carcinoid architecture; } \\
2-10 \text { mitoses/10 HPF; } \\
\text { focal necrosis }\end{array}$ & $\begin{array}{l}\text { CgA; synaptophysine; } \\
\text { NSE; expression of SR }\end{array}$ & $\begin{array}{l}\text { grade 2; } \\
\text { intermediate }\end{array}$ \\
\hline LCNEC & $\begin{array}{l}\text { neuroendocrine morphology (organoid nesting } \\
\text { palisading rosettes, trabeculae); large cell size } \\
\text { with low nuclear to cytoplasmatic ratio, vesicular } \\
\text { or fine chromatin; necrosis }\end{array}$ & $\begin{array}{l}\text { weak expression of synaptophysin; } \\
\text { weak expression of NSE; } \\
\text { P53 gene mutations }\end{array}$ & $\begin{array}{l}\text { grade } 3 \text {; } \\
\text { high }\end{array}$ \\
\hline SCNC & $\begin{array}{l}\text { small size; scant cytoplasm; nuclei: finely granular } \\
\text { chromatin, absent or faint nucleoli }\end{array}$ & $\begin{array}{l}\text { weak expression of synaptophysin; } \\
\text { weak expression of NSE; } \\
\text { P53 gene mutations }\end{array}$ & $\begin{array}{l}\text { grade } 3 ; \\
\text { high }\end{array}$ \\
\hline
\end{tabular}

HPF = High-power field; NSE = neuro-specific enolase; $S R=$ somatostatin receptor . 

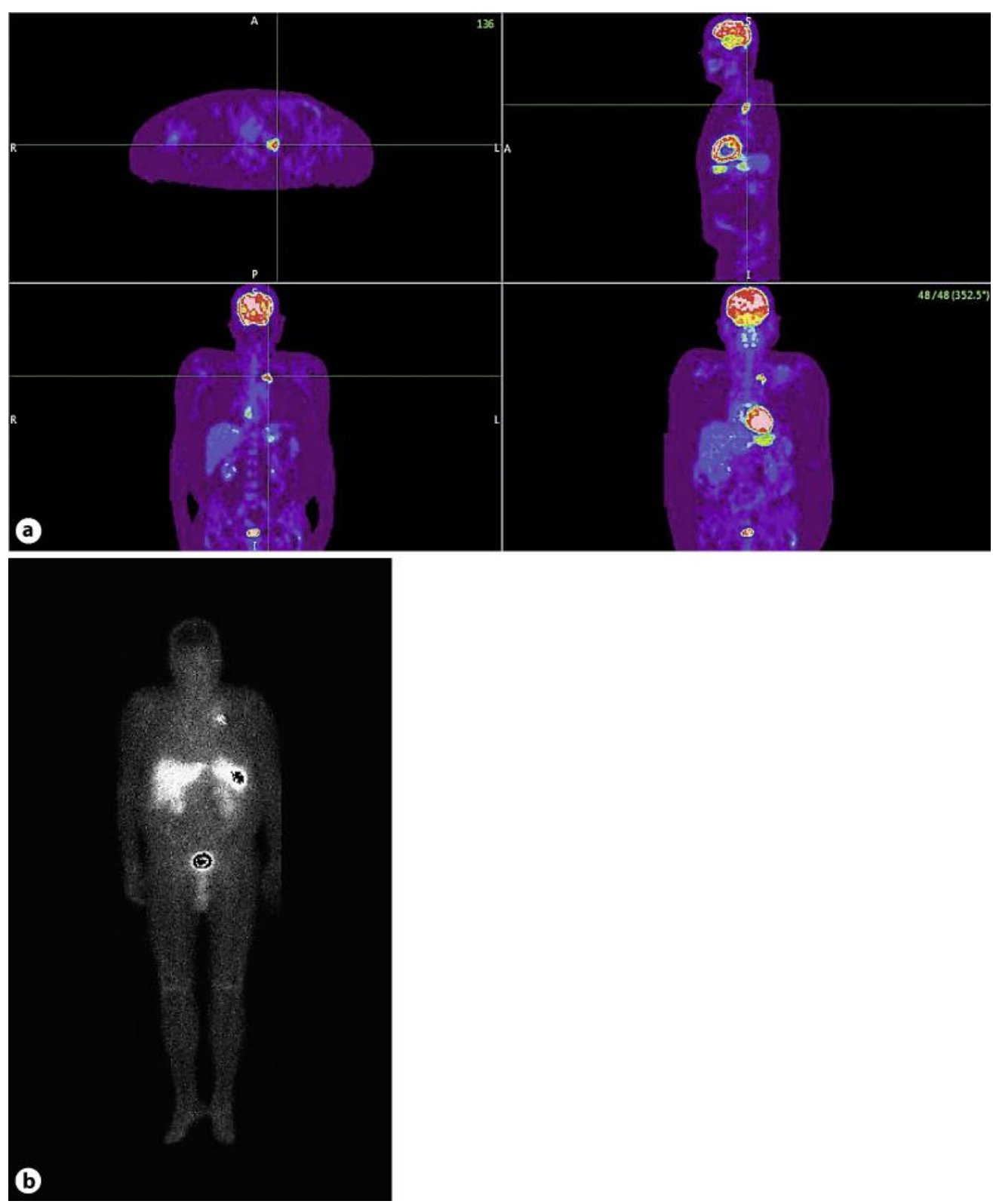

Fig. 1. Imaging at diagnosis: PET-CT (a) and octreotide scan (b). 

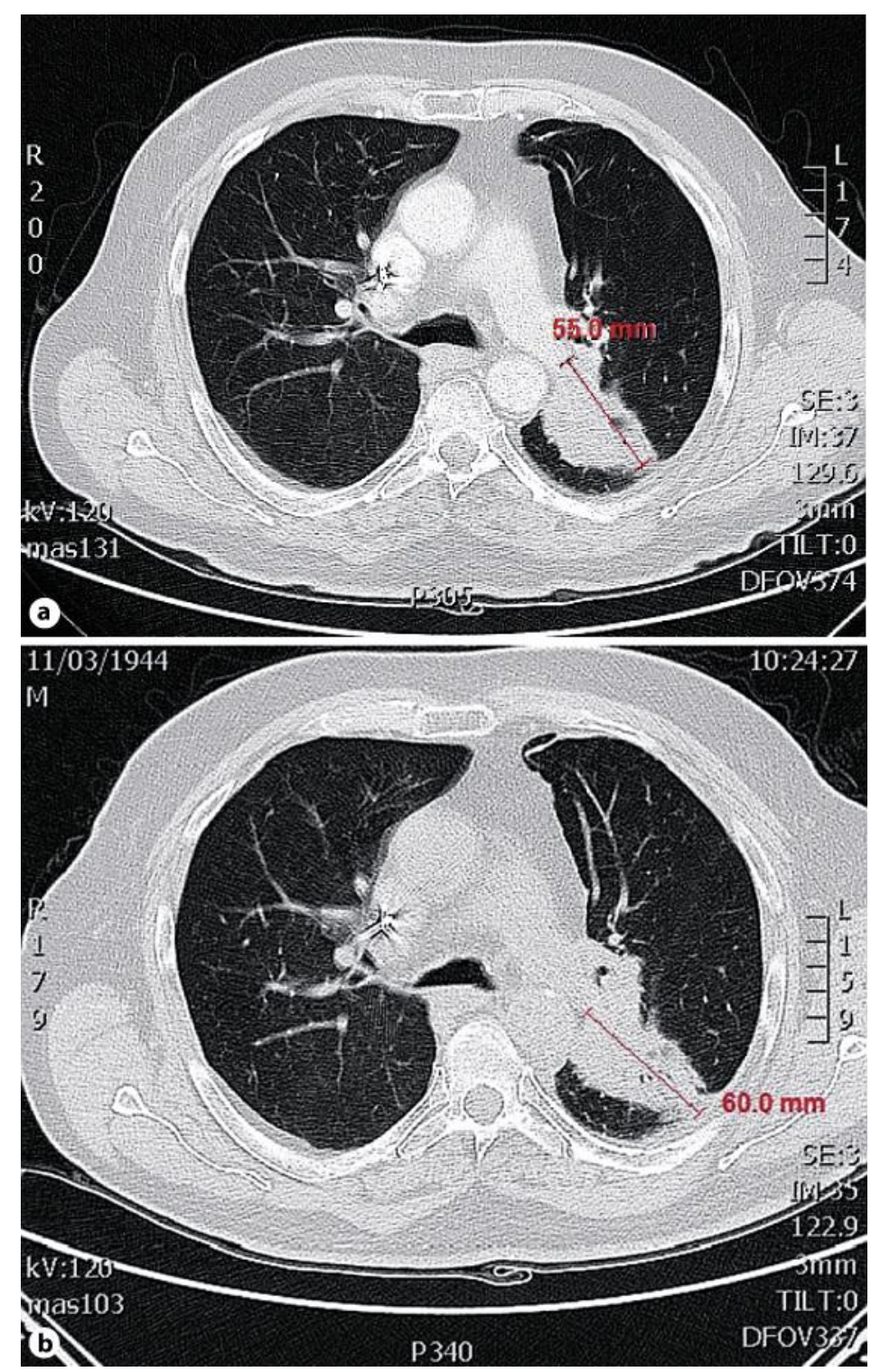

Fig. 2. Octreotide scan imaging at recurrence (a) and at the time of the patient's last visit (b). 

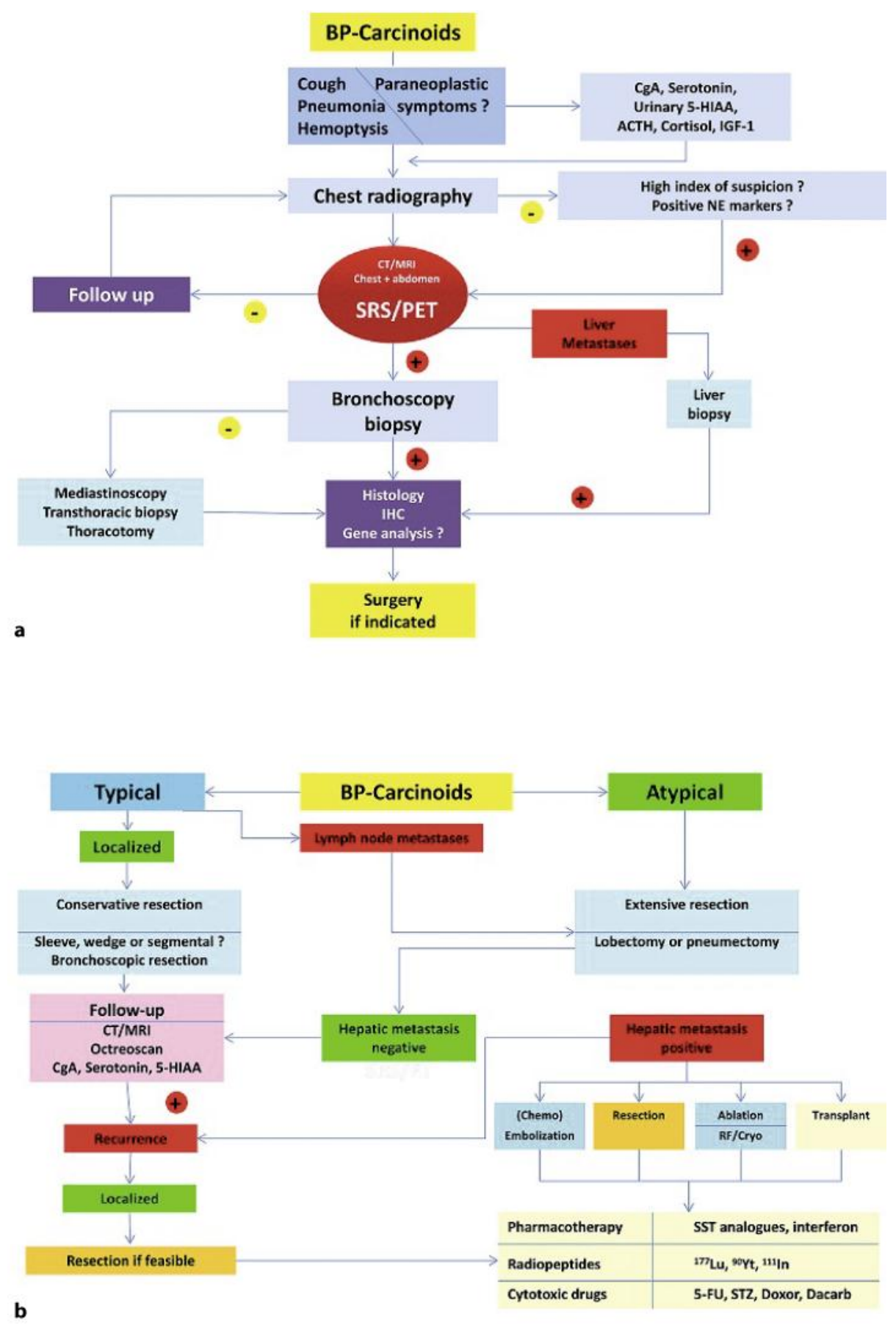

Fig. 3. Diagnostic (a) and treatment (b) algorithm for bronchopulmonary carcinoids. Taken from Bjorn et al. [7]. BP = Bronchopulmonary; ACTH = adrenocorticotropic hormone; IGF-1 = insulin-like growth factor 1; $\mathrm{NE}$ = neuron-specific enolase; $\mathrm{MRI}$ = magnetic resonance imaging; $\mathrm{SRS}$ = somatostatin receptor scintigraphy; $\mathrm{IHC}=$ immunohistochemistry; $\mathrm{RF}=$ radiofrequency; cryo = cryotherapy; SST = somatostatin; Lu = Lutetium; Yt = Yttrium; In = Indium; 5-FU = 5-fluorouracil; $\mathrm{STZ}=$ streptozotocin; Doxor $=$ doxorubicin; Dacarb $=$ dacarbazine. 


\section{References}

1 Modlin I, Lye K, Kidd M: A 5-decade analysis of 13,715 carcinoid tumors. Cancer 2003;97:934-959.

2 The US National Cancer Institute, Surveillance Epidemiology and End Results (SEER) data base, 19732004. http://seer.cancer.gov (accessed June 3, 2012).

-3 Eisenhauer EA, Therasse P, Bogaerts J, Schwartz LH, Sargent D, Ford R, Dancey J, Arbuck S, Gwyther S, Mooney M, Rubinstein L, Shankar L, Dodd L, Kaplan R, Lacombe D, Verweij J: New response evaluation criteria in solid tumours: revised RECIST guideline (version 1.1). Eur J Cancer 2009;45:228-247.

4 Caplin M, Yao JC: Handbook of Gastroenteropancreatic and Thoracic Neuroendocrine Tumours. Bristol, Bioscientifica, 2011.

5 Travis W: Advances in neuroendocrine lung tumors. Ann Oncol 2010;21(suppl 7):vii65-vii71.

6 6 Oberg K, Hellman P, Kwekkeboom D, Jelic S; ESMO Guidelines Working Group: Neuroendocrine bronchial and thymic tumours: ESMO clinical practice guidelines for diagnosis, treatment and follow-up. Ann Oncol 2010;21(suppl 5):v220-v222.

7 Bjorn I, Gustafsson M, Modlin I: Bronchopulmonary carcinoid tumors; in Modlin I, Oberg K (eds): A Century of Advances in Neuroendocrine Tumor Biology and Treatment. Hannover, Felsenstein CCCP 2007, pp 54-65.

8 Beasley M, Brambilla E, Travis W: The 2004 World Health Organization classification of lung tumors. Semin Roentgenol 2005;40:90-97.

9 Travis W, Brambilla E, Müller-Hermelink HK, Harris CC (eds): Pathology and Genetics of Tumors of the Lung, Pleura, Thymus and Heart. Lyon, IARC Press, 2004.

10 Kwekkeboom D, Krenning E, Scheidhauer K, Lewington V, Lebtahi R, Grossman A, Vitek P, Sundin A, Plöckinger U; Mallorca Consensus Conference participants; European Neuroen docrine Tumor Society: ENETS consensus guidelines for the standards of care in neuroendocrine tumors: somatostatin receptor imaging with (111)In-pentetreotide. Neuroendocrinology 2009;90:184-189.

11 National Comprehensive Cancer Network (NCCN) guidelines. www.nccn.org (accessed May 23, 2012).

12 O’Toole D, Grossman A, Gross D, Delle Fave G, Barkmanova J, O’Connor J, Pape UF, Plöckinger U; Mallorca Consensus Conference participants; European Neuroendocrine Tumor Society: ENETS consensus guidelines for the standards of care in neuroendocrine tumors: biochemical markers. Neuroendocrinology 2009;90:194-202.

13 Bianchi A, De Marinis L, Fusco A, Lugli F, Tartaglione L, Milardi D, Mormando M, Lassandro AP, Paragliola R, Rota CA, Della Casa S, Corsello SM, Brizi MG, Pontecorvi A: The treatment of neuroendocrine tumors with long-acting somatostatin analogs: a single center experience with lanreotide autogel. J Endocrinol Invest 2011;34:692-697.

14 ENETS guidelines: TNM grading, standard of care and metastasis. www.enets.org (accessed May 23, 2012).

15 Kidd M, Drozdov I, Joseph R, Pfragner R, Culler M, Modlin I: Differential cytotoxicity of novel somatostatin and dopamine chimeric compounds on bronchopulmonary and small intestinal neuroendocrine tumor cell lines. Cancer 2008;113:690-700. 